\title{
Spoofing Detection for Fingerprint using Hog and Iris using Surf Descriptor
}

\author{
Lekshmy S Mohan', Joby James ${ }^{2}$ \\ PG Student, College of Engineering Kidangoor, Kotttayam, A.P.J. Abdul Kalam Technological University ${ }^{1}$ \\ Asst Prof, Dept Electronics and Communication, College of Engineering Kidangoor ${ }^{2}$
}

\begin{abstract}
Now a days for security applications like identification and verification Biometric authentication systems are used. Biometric features are unique in nature, so this can be used to avoid typical problems of the system based on the use of password which can be stolen or forgotten. The research in this field is very active with local descriptors based on analysis of micro textural features in order to keep good level of security, gaining more and more popularity because of their excellent performance and flexibility. And this paper aims for accessing these descriptors for liveness detection in security systems based on various biometric traits: fingerprint, iris and face. In this work implements and evaluate two different feature extraction techniques for fingerprint and iris detection: - HOG and SURF descriptor respectively. Both the techniques were used in conjunction with a SVM classifier.
\end{abstract}

Keywords: HOG, LBP, SURF, SVM.

\section{INTRODUCTION}

Biometric is a rapidly developing technology to identify a person based on his or her physiological or behavioural characteristics .In order to ensure the correction of authentication, the biometric system must be able to detect and reject the use of copy of biometric instead of the live biometric. And this function is termed as "liveness detection". Biometric has the capability to distinguish between fake and real. Fingerprint ,face and iris are the biometric traits most frequently used in present security systems. Fingerprint system can be fooled by reproducing the biometric patterns on molds made of materials like silicone ,Play-Doh, clay or gelatine.

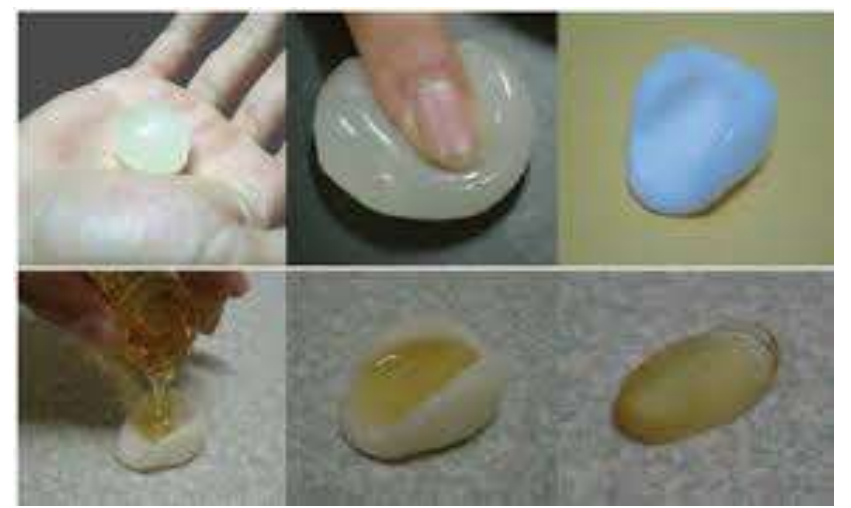

Fig 1.1 : Making an artificial fingerprint directly from live finger plastic , used to obtain the mold and gelatin to obtain the cast.

And iris based systems can be faked with wearable plastic lens or by using fake irises printed on paper.

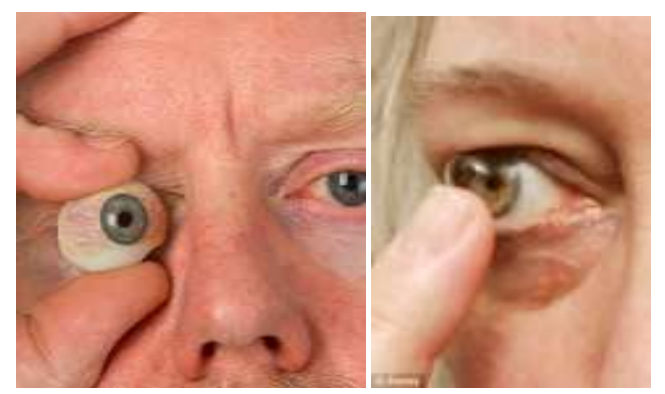




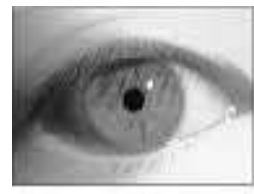

(a)

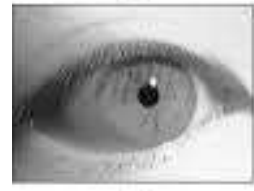

(6)

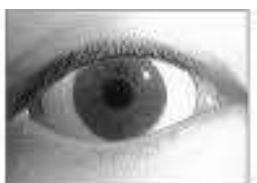

\$c)

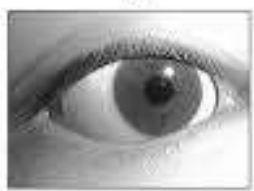

(d)

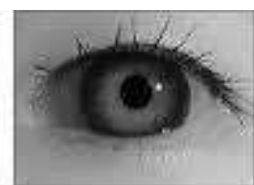

(s)

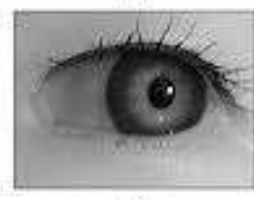

in

Fig 1.2: Wearable plastic lens and fake irises printed on paper.

In parallel to the approaches based on global and high level image descriptors techniques related on local descriptors have been taking hold recently [1] [2]. Local descriptors describe the statistical behaviour observed locally in very small patches of the image by means of histogram. And by means of conventional classification tools these histogram are used as features to classify the image. For image classification problem like image mining and retrieval, face recognition, image quality assessment local descriptors based approach is used.

Various fingerprint liveness detection algorithms have been proposed [3] [4] [5] and they can be broadly divided into two approaches; Hardware and software. In the hardware approach a specific device is included to the sensor in order to detect the properties of a living trait such as the blood pressure [6], skin distortion [7] or the odor [8].The main disadvantage of these methods is that they require extra hardware, which is expensive, bulky and not convenient to the users. In the software approach which is used in this work, fake traits are detected once the sample has been acquired with a standard sensor. The features used to distinguish between real and fake finger are extracted from the image of the fingerprint. These are techniques such as in [9] [10] in which the features used in the classifier are based on the specific fingerprint measurements such as ridge, strength, continuity and clarity.

Though iris features are unique, recent research results suggest that they are affected by several covariates such as pupil dilation [11] and sensor interoperability [12] [13]. Another factor that may affect the presence of transparent (soft) and colour cosmetic (textured) contact lenses.

Research in this field are active with extracting the features of local descriptors based on the micro-textural features like Local Binary Pattern. Histogram of Oriented Gradient (HOG) is one of the well known features for object recognition. N. Dala et al proposed an object detection algorithm in which HOG features were extracted from all locations of a dense grid on a image region and the combined features are classified by using Support Vector Machine. Iris serves one of the excellent biometric traits due to the stability and randomness of its unique features. Iris after localization, scale invariant feature transform is used to extract the local features. Since SIFT is found out to be computational complex. So in this paper another key point descriptor Speeded Up Robust features are used which gives promising results with less computations

\section{LITERATURE SURVEY}

For the liveness detection task in authentication systems based on various biometric traits like iris, face and fingerprint liveness spoofing detection is used. There are a lot of fake attempts will be detected in the authentication field. Various researches and techniques are used to reduce the error occurred in the detection field.

One of the first efforts in fingerprint liveness detection was carried out by [14] who initiated a research line using the skin perspiration pattern. And in a subsequently work [15], they used a wavelet based algorithm improving the performance reached in their initial study and yet in further step [16],they extended both works with a new intensity which is based on perspiration liveness detection techniques which leads to detection rates which is around $90 \%$ on a database .

In some research in parallel with skin elasticity, a liveness detection procedure which is based on the corporal order[17] use a chemical sensor o discriminate the skin odour from the materials such as gelatine or silicon.

Other liveness detection for fake fingerprint detection includes the analysis of perspiration and elasticity and the use of electric properties of skin[18],the use of the power spectrum of the fingerprint image [19] and using wavelets for analysis of the fingerprint image[20].

In some sophisticated techniques which use texture descriptors as feature vectors, such as load phase quantization [21], LBP with wavelets [22] and BSIF [23] use the original and uniform LBP coding schemes Outside the research field some companies proposed various methods for fingerprint liveness detection such as ultrasound [24] on electrical measurements or light measurements. 
Some techniques for the detection of biometric spoofing are based on the Local Binary Pattern, a descriptor first proposed in 2002 [25]for texture classification. The first iris recognition system was developed by Daugman at University of Cambridge. Daugman [26] has used multi scale quadrature wavelets to extract texture phase structure information of the iris in order to generate a 2048 bit iris code and compared the difference between a pair of iris representations by computing their Hamming distance via the XOR.

In 2009 [27] proposes the use of LBP to detect iris spoofing based on contract lens, where the iris is divided into six sub regions, these are rectified and LBPs are extracted at various scales. And finally the Adaboost algorithm is used to learn the most discriminative regional LBP features . More recently [28] divides the eye image into three regions; pupil, iris and sclera ,using LBP's at multiple scales for each of them.

In 2003 Daugman [29] proposed using Fourier transform to detect fake iris pattern that were prevalent in textural lenses manufactured at that time. Newer lenses however , have multiple layers of printing ,making the Fourier responses less and co-occurrence matrix. CASIA and BATH databases for genuine iris images and a special acquisition for textural contact lenses were used to construct two class-balanced datasets.

\section{PROPOSED SYSTEM}

The problem for liveness detection can be divided into two class, where an input fingerprint and iris image as to be assigned to one of two classes real and fake.

In this work the input fingerprint and iris images may not be clean ie it may contain noise. A simple median filter is used to remove this kind of salt and pepper type of noise.

And later histogram equalization for fingerprint and iris images are done .Histogram equalization is a technique used for adjusting the image intensities o enhance contrast. This method increases the global contrast of many images, when the usable data of the image is represented by close contrast values. And by this, the intensities can be distributed on the histogram. This will allows the lower contrast areas to gain higher contrast. Histogram equalization accomplishes this by spreading out the most frequent intensity values. This method is useful in images which have background and foregrounds that are both bright or both dark.

And for feature extraction in fingerprint and iris images Local Binary Pattern (LBP) is used. LBP are the local texture descriptor that have performed well in computer vision applications which includes texture classification and segmentation, image retrieval etc.

In its original version, the LBP assigns a label to every pixel of an image by thersholding each 8 neighbors of the $3 \times 3$ neighborhood with the center pixel value and considers the result as a unique 8 bit code represents the 256 possible neighborhood combinations.

Let $\mathrm{X}$ be the generic pixel and $\eta \mathrm{i}(\mathrm{X})$ the $\mathrm{i}^{\text {th }}$ of $\mathrm{P}$ neighbor which is sampled uniformly on a circle of radius $\mathrm{R}$ centered on $\mathrm{X}$. The basic features used in LBP are simply directional differences.

$F_{i}(X)=I(X)-I\left(\eta_{i}(X)\right)$

These features are quantized independently with a fixed two-level symmetric quantizer obtaining the indexes. A string of bits is represented synthetically by the integer.

$$
\mathrm{C}(\mathrm{X})=\sum_{\mathrm{i}=0}^{p-1} \operatorname{Ci}(X) 2^{\mathrm{i}}
$$

In the basic version of LBP these quantities are on subjected to further processing, which leads to feature vector length $2^{\mathrm{P}}, \mathrm{h}=$ hist $(\mathrm{C})$ where

$$
\mathrm{h}(\mathrm{i})=\sum_{X} \delta(C(X)-i)
$$

The concept of uniform patterns are introduced to reduce the number $f$ possible bins. If the binary pattern consists of at most two bitwise transitions from 0to 1 or vice versa then the LBP pattern is called as uniform.

\section{- $\quad$ Fingerprint}

When LBP is combined with Histogram of Oriented Gradient descriptor, it improves the detection performance considerably on some datasets. HOG is one of the well known features for object recognition.HOG features are calculated by taking orientation histograms of edge intensity in a local region.

The implementation HOG descriptors can be obtained by dividing the image into small connected regions called cells and for each cell compiling a histogram of gradient directions or edge orientations for the pixels within the cell

The magnitude of the gradient is :- $|\mathrm{G}|=\sqrt{\mathrm{I}^{2} \mathrm{x}+\mathrm{I}^{2} \mathrm{y}}$.

The orientation of the gradient is :- $\theta=\arctan \frac{\mathrm{Iy}}{\mathrm{Ix}}$.

For the changes in illumination and contrast, the gradient strengths must be locally normalized, which requires the grouping of cells together into large. 
The HOG descriptor is then the vector of the components of the normalized cell histograms from all of the block regions.

L1-norm: $f=\frac{v}{(\|v\| 1+e)}$
L2-norm: : $f=\frac{v v}{(\|v\| 2+e 2)}$

\section{- Iris}

Here for iris image Surf descriptor is used for feature extraction as just like HOG descriptor used for fingerprint feature extraction. Surf technique is used for feature extraction and matching purpose. For feature extraction normalized iris region is considered. And SURF provides the following steps:-

a. Blob detector based on Hessian Matrix is used to detect interest points.

The locations where the Hessian determinant is maximum gives rise to an interest point.

The Hessian matrix as given as follows:

$$
\mathrm{H}(\mathrm{x}, \sigma)=\left[\begin{array}{ll}
\operatorname{Lxx}(\mathrm{x}, \sigma) & \operatorname{Lxy}(\mathrm{x}, \sigma) \\
\operatorname{Lxy}(\mathrm{x}, \sigma) & \operatorname{Lyy}(\mathrm{x}, \sigma)
\end{array}\right]
$$

Where $H(x, \sigma)$ is the Hessian matrix at $x$ a scale $\sigma$.

The determinant of a hessian matrix expresses the extent of the response and is an local change around the area .

expression of the

b. Based on information from a circular region around the interest point fix a reproducible orientation. Then construct a square region aligned to the selected orientation and after that extract the SURF descriptor form it.

c. Matching pairs can be found by comparing the descriptors obtained from processing image and already stored image.

d. The smallest Euclidean distance between the same points represents a good match.

As the final step for classification SVM classifier is used for iris and fingerprint image. Through classification it is possible to distinguish between real and fake.

SVM classifier is used to processing, such classifier can be used for texture analysis. A special property of SVM is that it can minimize the empirical classification error and maximize the empirical margin. So SVM called Maximum Margin Classifier.SVM maps the input vector to a higher dimensional space where a maximal separating hyper plane is constructed. Two parallel hyper plane are constructed on each side of the hyper plane that separate the data. The separating hyper plane is the hyper plane that maximize the distance between the two parallel hyper planes.

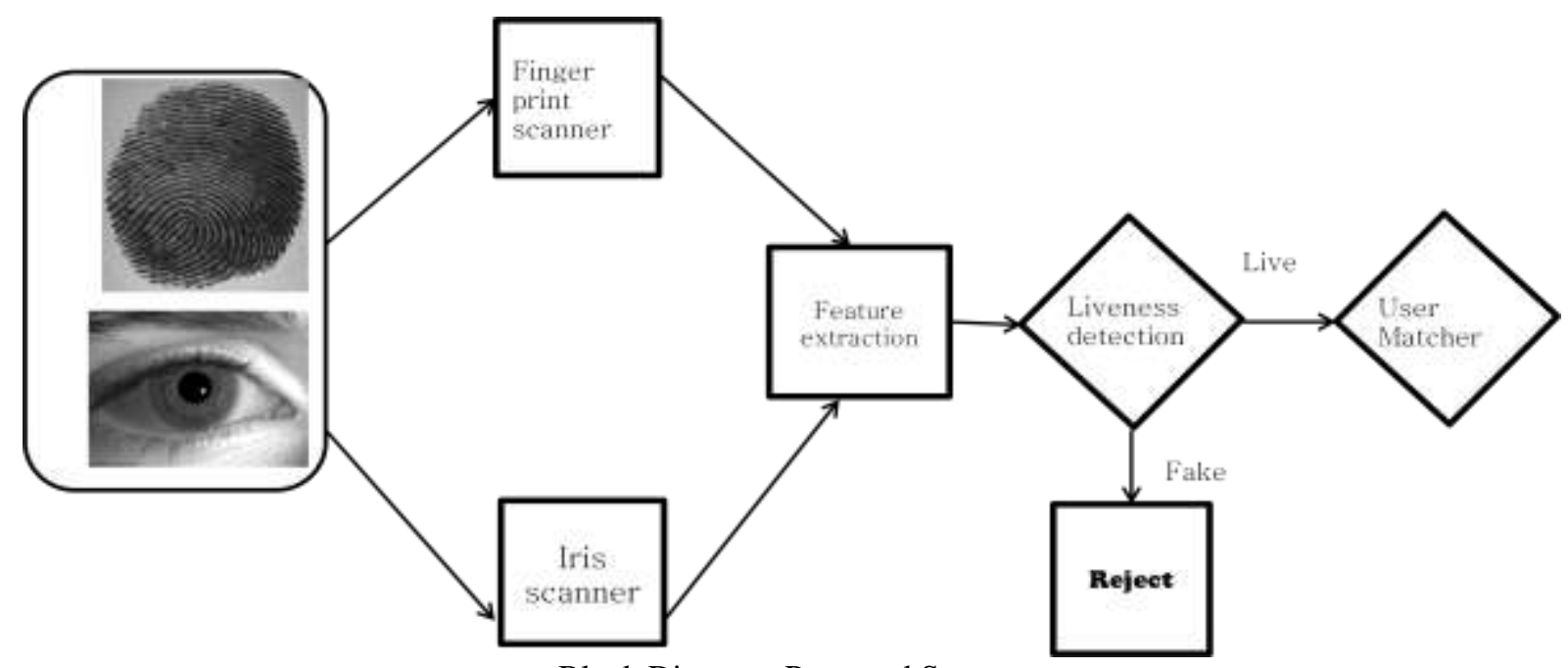

Block Diagram: Proposed System

\section{EXPERIMENTAL RESULTS}

Simulation procedure is carried in the MATLAB domain. The effectiveness of the proposed spoofing detection strategy is ascertained using experimental results on Clarkson University spoof and real iris and fingerprint database. And performed experiments on the databases of iris and fingerprint .MATLAB analysis shows the pictorial representation of spoof detection of iris and fingerprint. 
- $\quad$ FINGERPRINT

A.ANALYSIS OF SPOOF FINGERPRINT IMAGE

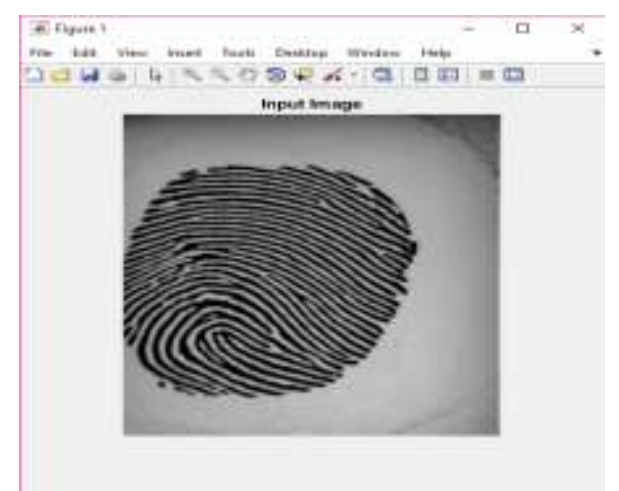

Fig 4.1: Input image

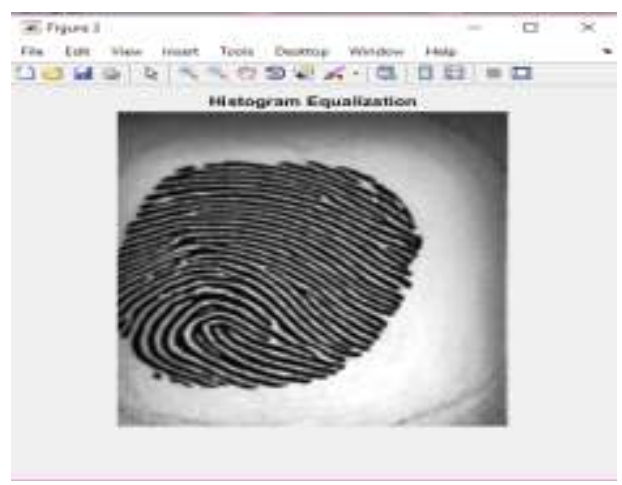

Fig 4.2: Histogram Equalization

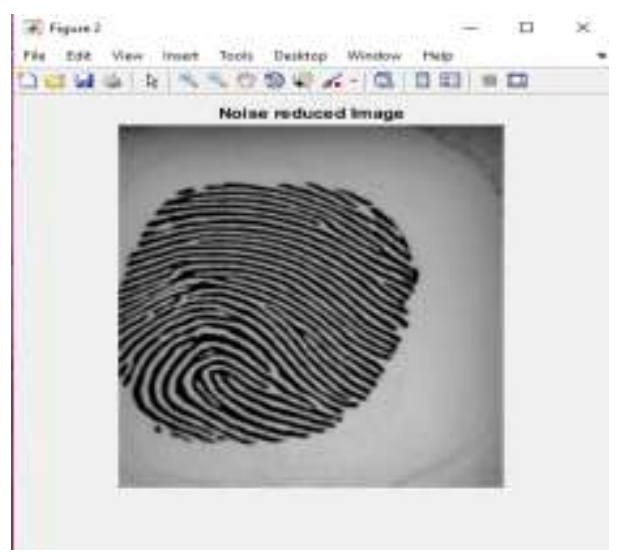

Fig 4.3 Noise reduced

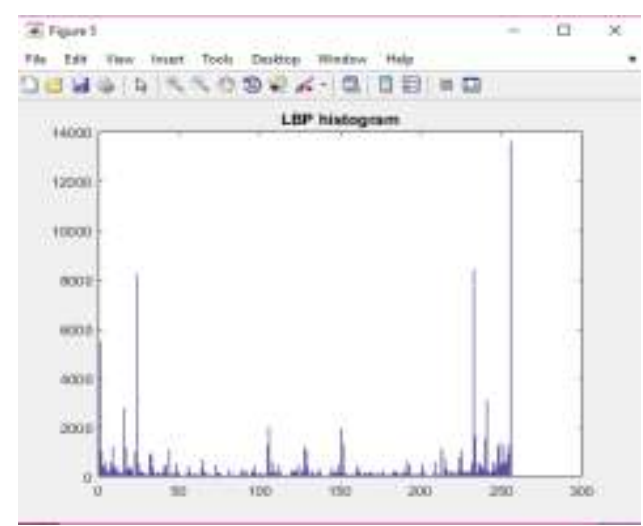

Fig 4.4: LBP histogram 
International Journal of Advanced Research in Computer and Communication Engineering ISO 3297:2007 Certified

Vol. 6, Issue 4, April 2017

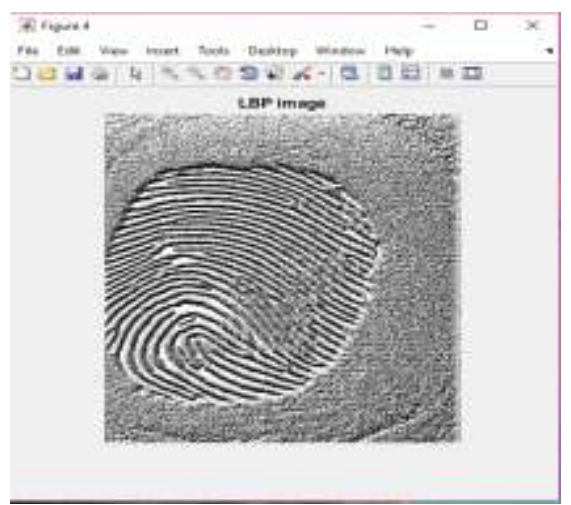

Fig 4.5: LBP image

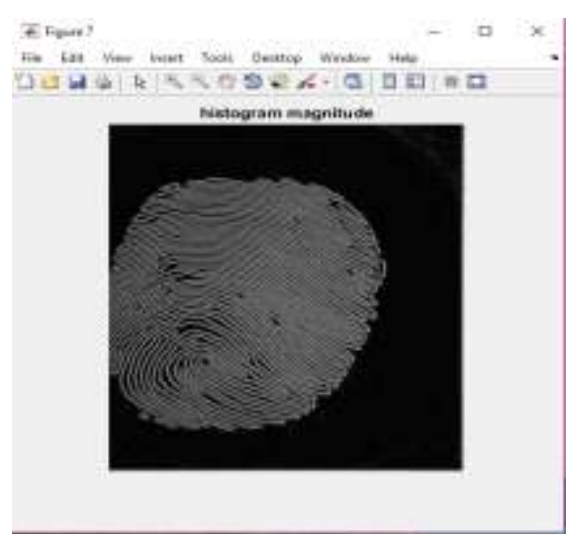

Fig 4.6: Histogram magnitude

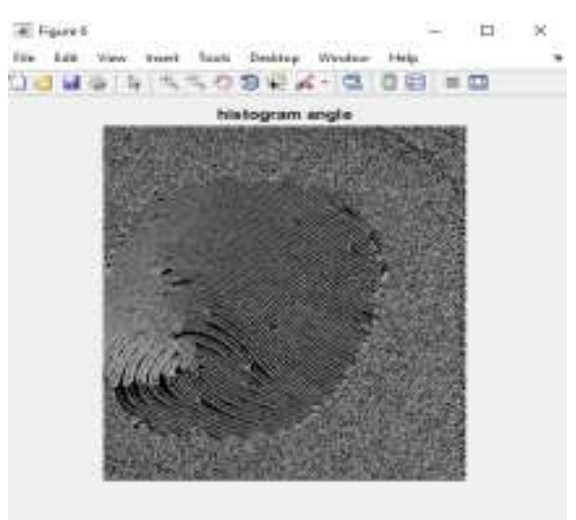

Fig 4.7: Histogram angle

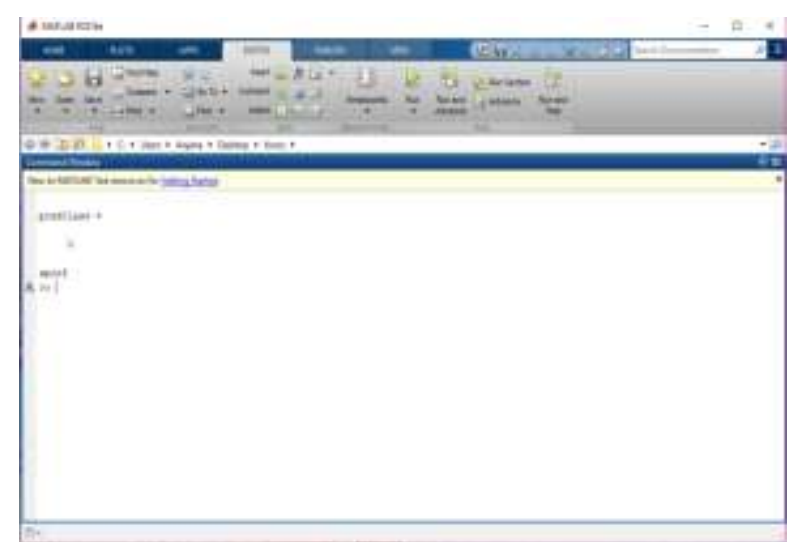

Fig 4.8: Command window showing that input fingerprint image is spoof 
-

IRIS

By using MATLAB test image is compared with reference image and ten strongest points are detected using SURF descriptor. And finally only for real image matched points are founded.

B. ANALYSIS OF SPOOF IRIS IMAGE

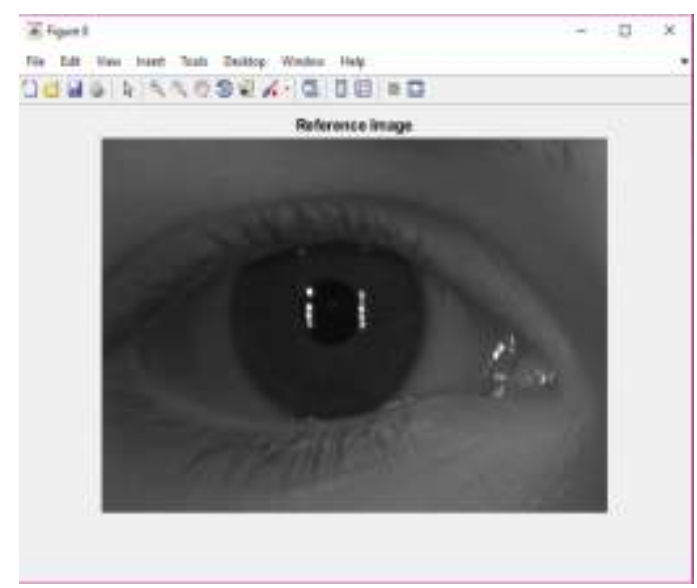

Fig 4.9: Reference image

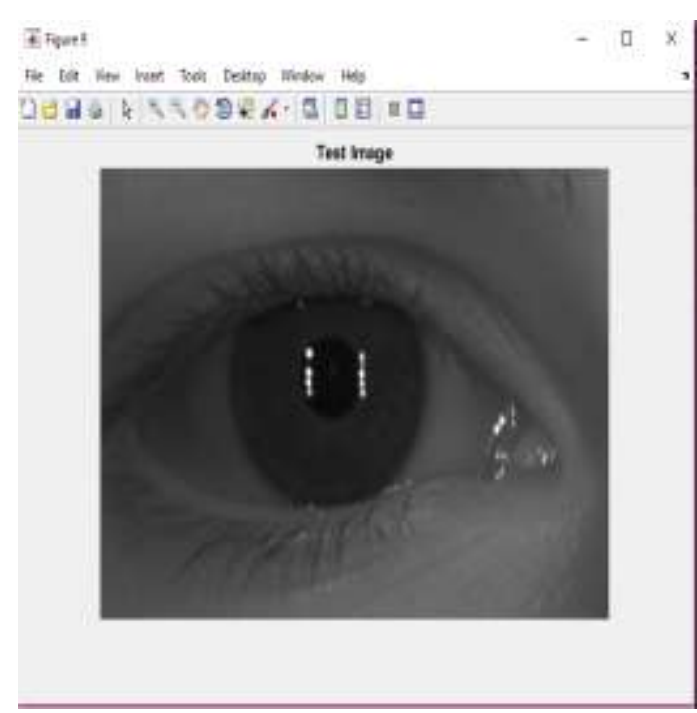

Fig 4.10 : Test image

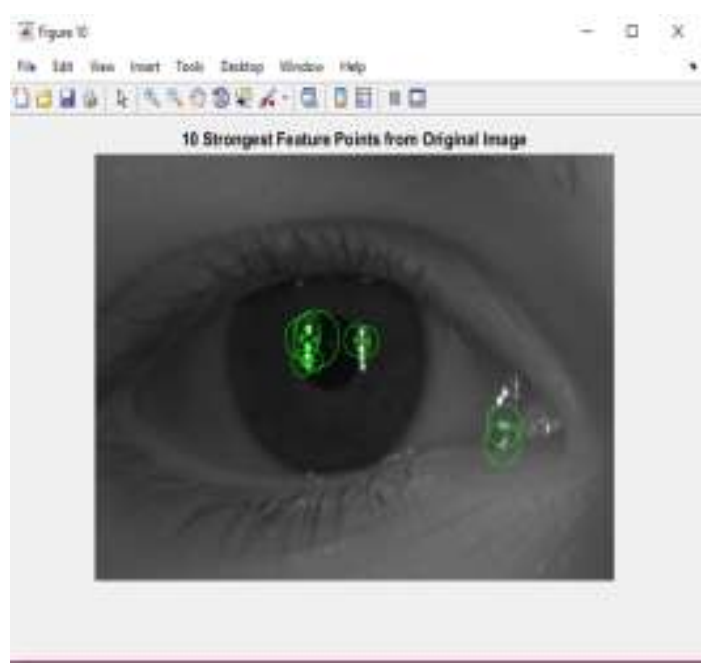

Fig 4.11: Strongest feature points from original image 


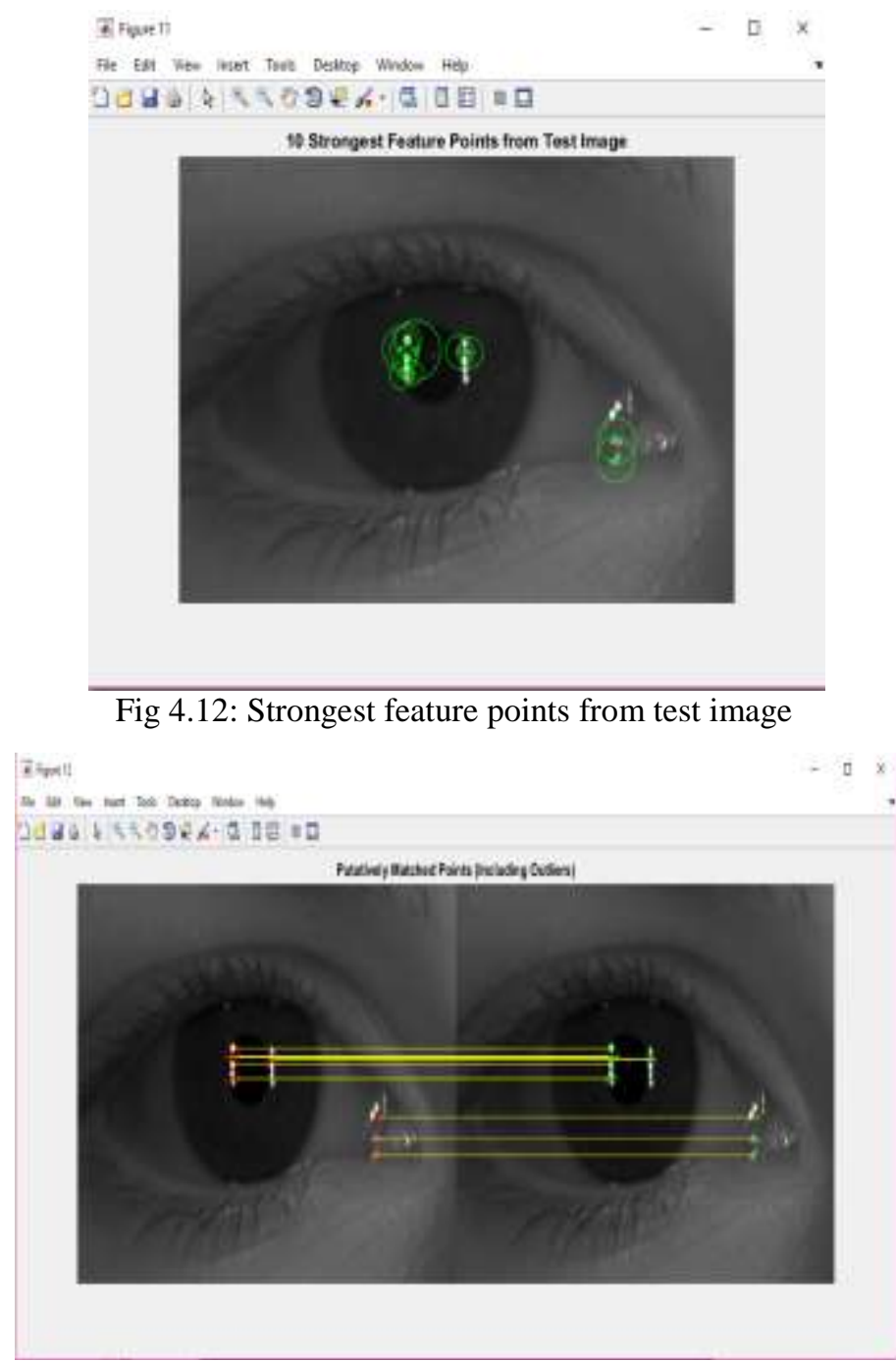

Fig 4.13: Putatively Matched Points

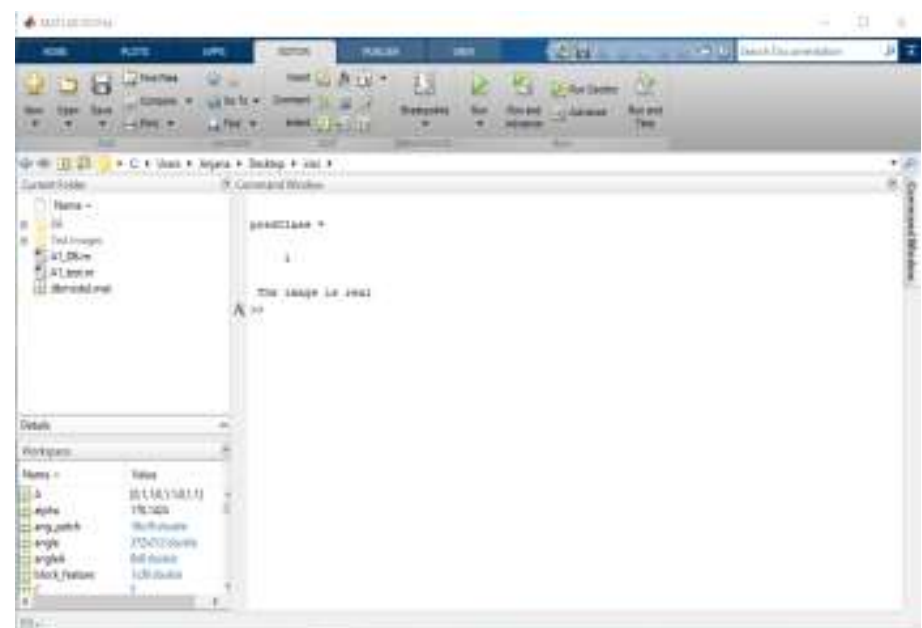

Fig 4.14: Command window showing input iris image is real

\section{CONCLUSION}

From the analysis we can identify the real and spoof imagesof fingerprint and iris images by perfroming Histogram of Oriented Gradient and Surf descriptor respecively.In this work analytical and stimulation of the images are done. Conventional methods for detection of liveness in a fingerprint image of a user included: Temperature detection, 
measurement of pulse, incorporation of the concept of pulse oximetry, electrocardiogram and detection of perspiration. Since the aforementioned methods turned out to be expensive, software methods are much preferred . The work is carried out in MATLAB domain which reduces hardware complexity and cost. The advantage of using SURF is that it shows a higher robustness. The standard version of SURF is several times faster than SIFT. The essential thought behind the histogram of oriented gradient descriptor is that local object appearance and shape within an image can be described by the distribution of intensity gradients or edge directions. Through this proposed system we can keep a good level of security.

\section{REFERENCES}

[1] S. B. Nikam and S. Agarwal, "Local binary pattern and wavelet based spoof fingerprint detection,” Int. J. Biometrics, vol. 1, no. 2,pp. 141-159, 2008.

[2] N. Erdogmus and S. Marcel, "Spoofing face recognition with 3D masks,”IEEE Trans.Inf. Forensics Security, vol. 9, no. 7, pp. 1084-1097,Jul. 2014.

[3] Y. Chen, A. Jain, and S. Dass, "Fingerprint deformation for spoof detection," in Proc. Biometric Symp., 2005 , p. 21.

[4] B. Tan and S. Schuckers, "Comparison of ridge- and intensity-based perspiration liveness detection methods in fingerprint scanners," Proc. SPIE, vol. 6202, p. 62020A, Apr. 2006.

[5] P. Coli, G. L. Marcialis, and F. Roli, "Fingerprint silicon replicas: Staticand dynamic features for vitality detection using an optical capturedevice,” Int. J. Image Graph., vol. 8, no.4, pp. 495-512, 2008.

[6] P. D. Lapsley, J. A. Lee, D. F. Pare, Jr., and N. Hoffman, "Anti-fraud biometric scanner that accurately detects blood flow," U.S. Patent 5737 439, Apr. 7, 1998.

[7] A. Antonelli, R. Cappelli, D. Maio, and D. Maltoni, "Fake finger detection by skin distortion analysis," IEEE Trans. Inf. Forensics Security, vol. 1 , no. 3, pp. 360-373, Sep. 2006.

[8] D. Baldisserra, A. Franco, D. Maio, and D. Maltoni, "Fake fingerprint detection by odor analysis," in Advances in Biometrics. Heidelberg, Germany: Springer, 2005, pp. 265-272.

[9] V. Mura, L. Ghiani, G. L. Marcialis, F. Roli, D. A. Yambay, and S. A. Schuckers, "Livdet 2015 fingerprint liveness detection competition 2015," in Proc. IEEE 7th Int. Conf. Biometrics Theory, Appl. Syst., Sep. 2015, pp. 1-6.

[10] D. Gragnaniello, G. Poggi, C. Sansone, and L. Verdoliva, "Fingerprint liveness detection based on weber local image descriptor," in Proc. IEEE Workshop Biometric Meas. Syst. Secur. Med. Appl. (BIOMS), Sep. 2013,pp. 46-50.

[11] K. Hollingsworth, K. W. Bowyer, and P. J. Flynn, "Pupil dilation degrades Iris biometric performance," Comput. Vis. Image Understand., vol. 113, no. 1 , pp. $150-157,2009$.

[12] S. S. Arora, M. Vatsa, R. Singh, and A. K. Jain, “On Iris camera interoperability,” in Proc. BTAS, 2012, pp. 346-352.

[13] R. Connaughton, A. Sgroi, K. Bowyer, and P. Flynn, "A multi algorithm analysis of three Iris biometric sensors," IEEE Trans. Inf. ForensicsSecurity, vol. 7, no. 3, pp. 919-931, Jan. 2012.

[14] R. Derakhshani, S. Schuckers, L. Hornak, L. O'Gorman, Determination of vitality from non-invasive biomedical measurement for use in fingerprint scanners,Pattern Recognition 36 (2003) 383-396.

[15] S. Schuckers, A. Abhyankar, A wavelet based approach to detecting liveness in fingerprint scanners, in: Proc. Biometric Authentication Workshop (BioAW), in: LNCS, vol.5404, Springer, 2004, pp. 278-386.

[16] B. Tan, S. Schuckers, Comparison of ridge- and intensity-based perspiration liveness detection methods in fingerprint scanners, in: Proc. SPIE Biometric Technology for Human Identification III, BTHI III, vol. 6202, 2006, 62020A.

[17] O.G.Martinsen,S.Clausen,J.B.Nysather,S.Grimmes,Utilizing characteristic electrical properties of the epidermal skin laers o deect fake fingers in biometric fingerprint systems-a pilt study,IEEE Transactions on Biomedical Engineering 54(2007)891-894.

[18] P.Coli,G.I.Marcialis,F.Roli,Power spectrum based fingerprint vitality detection,in:Proc.IEEE Workshop on Automatic Identificatin Advanced Technologies,AutID,2007,pp.169-173.

[19] Y.S.Mon,J.S.Chen,K.C.Chan,K.S,K.C.Woo,Wavele based fingerprint liveness detection,Electrnic Letters 41 (2005) $1112-1113$.

[20] Y.S.Mn.J.S.Chen.K.C.Chan,K.So.K.C.Woo,Wavelet based fingerprint liveness detection, Electronic Letters 41(2005)1112-1113.

[21] L. Ghiani, G. L. Marcialis, and F. Roli, "Fingerprint liveness detection by local phase quantization," in Proc. 21st Int. Conf. Pattern Recognit. (ICPR), 2012, pp. 537-540.

[22] L. Ghiani, G. L. Marcialis, and F. Roli, "Fingerprint liveness detection by local phase quantization," in Proc. 21st Int. Conf. Pattern Recognit. (ICPR), 2012, pp. 537-540.

[23] L. Ghiani, A. Hadid, G. L. Marcialis, and F. Roli, "Fingerprint liveness detection using binarized statistical image features," in Proc. IEEE 6th Int. Conf. Biometrics, Theory, Appl. Syst. (BTAS), Sep./Oct. 2013, pp. 1-6.

[24] Ultra-scan,2009.http://www.ultra-scan.com/.

[25] T. Ojala, M. Pietikäinen, and T. Mäenpää, "Multiresolution gray-scale and rotation invariant texture classification with local binary patterns," IEEE Trans. Pattern Anal. Mach. Intell., vol. 24, no. 7, pp. 971-987, Jul. 2002.

[26] P. Gupta, H. Mehrotra, A. Rattani, A. Chatterjee and A.K. Kaushik. "Iris recognition using corner detection," 23rd International Biometric Conference, Montreal, Canada, 2006

[27] Z. He, Z. Sun, T. Tan, and Z. Wei, "Efficient iris spoof detection via boosted local binary patterns," in Advances in Biometrics, vol. 5558. Berlin, Germany: Springer-Verlag, 2009, pp. 1080-1090.

[28] D. Yadav, N. Kohli, J. S. Doyle, R. Singh, M. Vatsa, and K. W. Bowyer,"Unraveling the effect of textured contact lenses on iris recognition," IEEE Trans. Inf. Forensics Security, vol. 9, no. 5, pp. 851-862,May 2014.

[29] J. Daugman, "Demodulation by complex-valued wavelets for stochastic pattern recognition,” Int. J. Wavelets, Multiresolution Inf. Process.,vol. 1 , no. 1 , pp. 1-17, 2003.

[30] Diego Gragnaniello, Giovanni Poggi,” An Investigation of Local Descriptors for Biometric Spoofing Detection”. IEEE TRANSACTIONS ON INFORMATION FORENSICS AND SECURITY, VOL. 10, NO. 4, APRIL 2015. 